\title{
Enhancing product structure design for an automation mechanism, by involving axiomatic design theory
}

\author{
Vasile V. Merticaru*, Eugen Merticaru, Ioan Butnariu and Marius-Marian Cucos \\ "Gheorghe Asachi" Technical University of Iasi, Romania
}

\begin{abstract}
The research presented in the paper is part of a larger approach concerning the enhancing of Product Development Sustainability by involving advanced design theories for effectiveness and efficiency of Product Design and CAD/CAE/CAM activities. The particular idea is to apply some principles from the Axiomatic Design theory within Concept Design and System Level Design stages, in order to enhance the Product Structure Design and to streamline the CAD activities and to study how a decomposition model obtained by applying AD theory affects the capture of Design Intent into a 3D CAD parametric product model structure. An applied project is exemplified as a case study, particularly referring to developing the Product Structure Design activities for the mechanical structure of an automated dimensional sorting system. Related discussions, conclusions and some further research directions are included.
\end{abstract}

\section{Introduction. Research problem and idea statement}

There is over a quarter of a century since Suh has introduced the Axiomatic Design (AD) theory [1], as a general scientific basis for systematic designing of complex systems [2], practically applicable for any kind of product or process to be developed, no matter how complex it is [3]. As it has been shown by Brown [4], an essential aspect for effectively applying AD theory in New Product Development (NPD) is to think an appropriate decomposition process. Actually, AD can be used to solve any level of product or process design, starting from the Concept Design, getting through System Level Design and up to the Detailed Design phase, as defined by Kressy [5], depending on the in-depth of the decomposition approach. While some researchers, [6], primarily consider AD useful to be applied in the Concept Design phase, for being able to facilitate the selection of the most adequate product concept solution among various others, most of them have also studied the value of the theory within System Level Design, [1-3, 7, 8], for being able to provide a good product structure solution. Otherwise, several design philosophies, theories, methods and instruments have been identified to be used for solving Product Structure Design as a main stage in Product Design, [9], respectively for obtaining a sustainable product structure. Design Thinking [10], Holistic Design [11], Modular Product Design [12],

\footnotetext{
*Corresponding author: merticaru@tcm.tuiasi.ro
} 
Reconfigurable Product Design [13], Design for Excellence (DfX) [14] etc. have been nominated and discussed among them, $[9,15]$.

On the other hand, Sustainability of Product and Process Development has been investigated in direct relation with CAD activities effectiveness and efficiency [15] and, as a result, translating, adapting, implementing, combining and enhancing concepts, principles, strategies, methods and tools from IT industry into CAD activities have been proposed as a way for performance in CAD. The importance of delivering an optimized solution for a $3 \mathrm{D}$ CAD parametric product model within digital product development, as valuable instrument for sustainable design and for sustaining green production, has been demonstrated by studies developed upon CAE activities based on FEA analyses of complex geometry parts [16] and also upon optimization of CNC manufacturing [17], the last one concluding that $\mathrm{CNC}$ machining performance requires special adapted methods for CAM designing technologies and for data management inside manufacturing industries.

The particular idea of the hereby presented research is to apply some design principles coming from the Axiomatic Design theory within Concept Design and System Level Design stages from NPD, in order to enhance the Product Structure Design and to streamline the CAD activities and to study how a decomposition model obtained by applying $\mathrm{AD}$ theory affects the capture of Design Intent in a 3D CAD parametric product model structure. The bellow presented research is part of a larger approach concerning the enhancing of Product Development Sustainability by involving advanced design theories for effectiveness and efficiency of Product Design and CAD/CAE/CAM activities.

\section{Research approach substantiation. Conceptual model}

The following two research hypotheses have been considered:

H1: AD theory used within Concept Design and System Level Design stages from NPD should enhance the Product Structure Design and streamline the CAD activities.

H2: A decomposition model obtained by applying AD theory, by delivering a reliable modular FRs/DPs based product structure, can provide a robust solution for a 3D CAD parametric product model structure, in terms of appropriately capturing the Design Intent.

A conceptual model as in Figure 1 has been structured based on a detailed conceptual analysis. The research approach has followed the algorithm designated in this model.

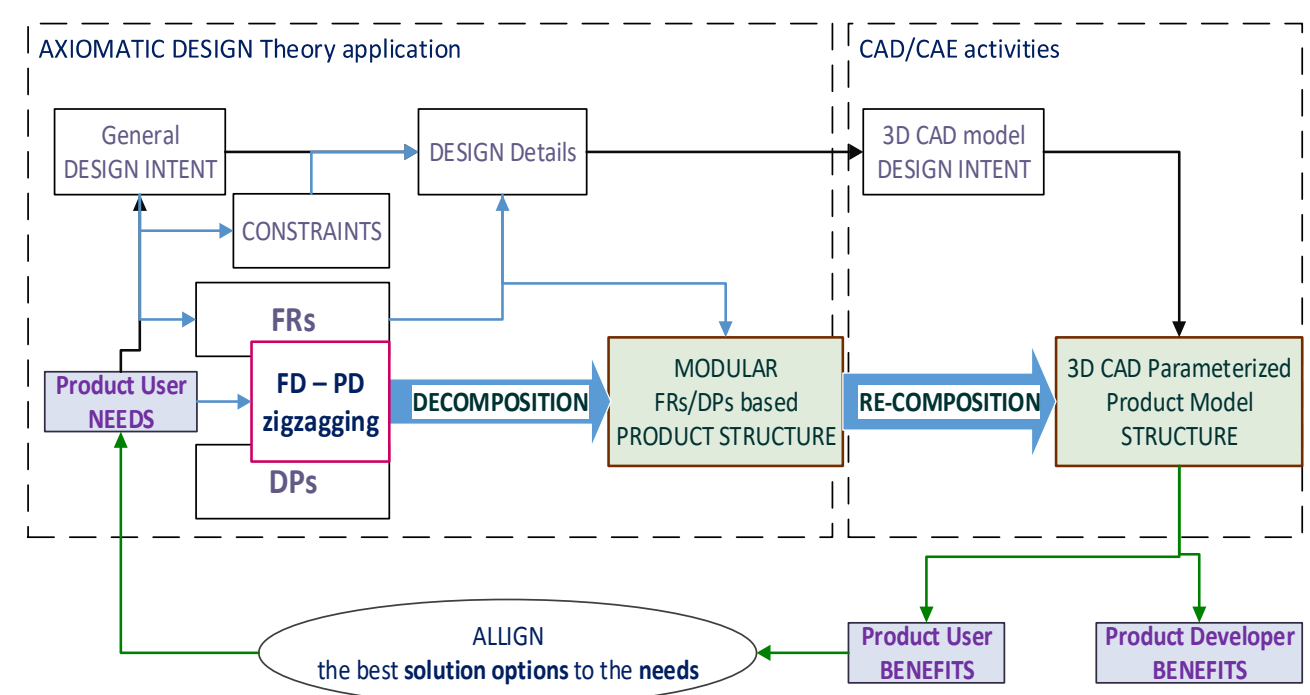

Fig. 1. Conceptual model for the research approach. 


\section{Case study. AD in mechanical structure design of an automation device}

An applied project is exemplified further on as a case study, particularly referring to developing the Product Structure Design activities for the mechanical structure of an automated dimensional sorting system. The choice of this kind of product to be studied stands on the similarities to AD theory of the classical design principles for automated manipulation systems which usually consider an overall automation function, its decomposing and further on finding a structure of technical solutions able to realize the identified set of sub-functions [18]. The applied study has particularly consisted in redesigning and reengineering, on new and advanced bases, of an older technical solution of such a mechanical automated device, [19], for having a comparison standard in terms of efficiency and effectiveness. The main steps in applying AD theory are briefly pointed here.

As customer needs $(\mathrm{CN})$, the following have been identified: $C N 1$ : the device could be achieved within an educational laboratory for mechanical automation, in order to illustrate automated sorting function; $C N 2$ : the device could realize automated dimensional sorting of a category of bearing rollers, in 3 diameter dimension classes; $C N 3$ : the equipment must ensure operating safety and constructive simplicity. As a constraint (Cs), the following has been considered: $C s 1$ : the device structure should contain as more as possible mechanical solutions for accomplishing the automation sub-functions. The general design intent has been defined by the main functional requirement FRO: Sort in 3 diameter dimension classes, a flow of bearing rollers, favorably oriented in vertical axis position; this has been put in correspondence with the main design parameter DPO: Automated dimensional sorting system. A decomposition model has been searched further on by zigzagging between the Functional Domain (FD) and the Physical Domain (PD) based mainly on objective oriented mapping of technical solutions as DPs (Design Parameters) to correspond to identified FRs (Functional Requirements), which has been accomplished considering general and particular functions and solutions existing in mechanical automation, [20], general theory of mechanisms, [21] and a synthesis algorithm of mechanisms according to a new concept with accent on the design integrated with new manufacturing paradigms, [22]. Some principles of DFD-DFR (Design for Disassembly and for Recycling) as defined by Crowther [23] and studied within a previous component of the above mentioned enlarged research, [24], also have been considered. An uncoupled design solution as in Figure 1 has been obtained, where the thick boxes represent the final state of the decomposition branches, respectively the model leaves.

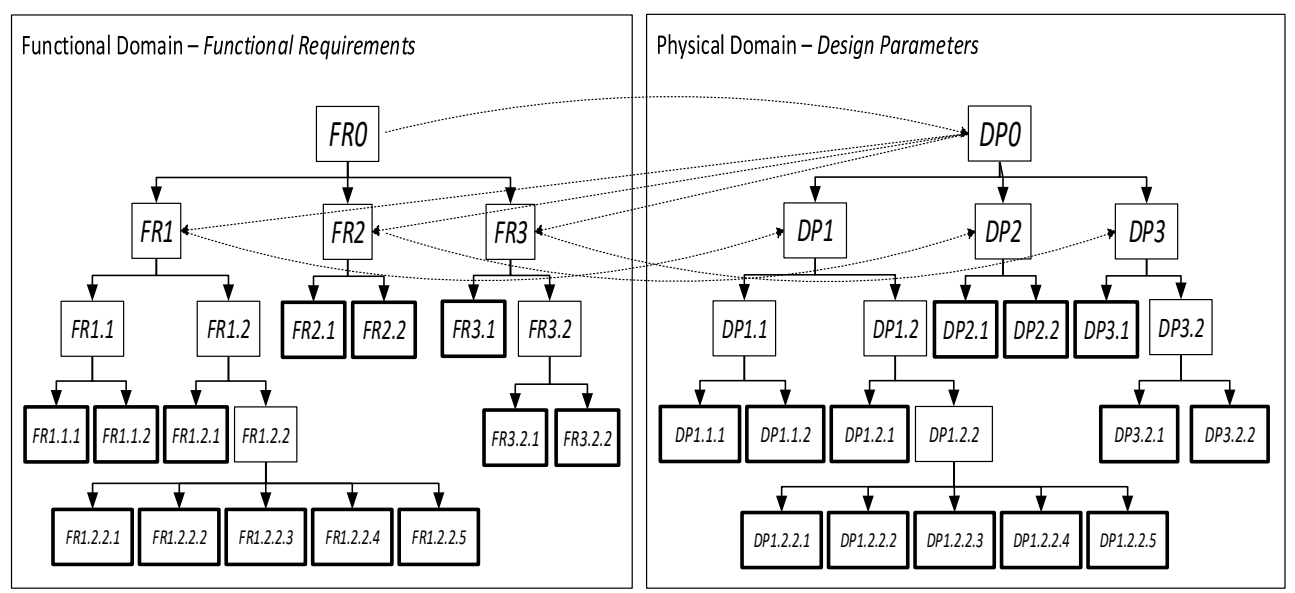

Fig. 2. Zigzagging decomposition model for the considered uncoupled design solution. 
In the model from Figure 1, the functional requirements (FRs) of first order are:

FR1: Feed the sorting system with temporization and transfer parts intermittently, maintaining part orientation and avoiding flow interruption (feed the measuring station with one by one parts having a favorable orientation and further on transfer them to selective evacuation station, in conditions of pre-established temporization and without flow interruption); FR2: Obtain and deliver the command parameter for sorting (measure the diameter dimension of the part arrived in the measuring station, compare the measured value with the two limit values parting the considered three dimensional ranges and deliver the command parameter); FR3: Sort the measured parts (Evacuate the measured part on selective direction, depending on the corresponding diameter range; Evacuate parts on selective directions).

In accordance with these, the following design parameters (DPs) of first order are: DP1: Automated feeding and transfer mechanism; DP2: Measuring station; DP3: Dimensional sorting station;

The defining of the FRs of second order is formulated as following:

FR1.1: Feed the system with a completely ordered line of favorably oriented parts, without flow interruption; FR1.2: Escape and place parts (from the feed track to the measuring station) (Separate one by one, favorably oriented parts from the completely ordered line and deliver them into the measuring station); FR2.1: Obtain the reaction parameter (input signal) (Measure the diameter dimension deviation of the part arrived in the measuring station); FR2.2: Process input signal into command parameter; FR3.1: Materialize the multiple evacuation way; FR3.2: Evacuate parts on selective directions.

In accordance with these, the following design parameters (DPs) of second order are: DP1.1: Gravity delivery feed track; DP1.2: Escape and part-placing system; DP2.1: Inductive transducer; $D P 2.2$ : Electronic block; $D P 3.1$ : 3 way linear gravity track; DP3.2: Gate escapement.

Targeting the detailed decomposition of mechanical structure, the defining of the FRs of third order is formulated as following:

FR1.1.1: Feed the system with favorably oriented parts (Feed the system with a partially ordered line of favorably oriented parts (not equidistant)); FR1.1.2: Accumulate an equidistant row of favorably oriented parts (Bring in completely order the line of favorably oriented parts by accumulating them equidistantly); FR1.2.1: Catch the part for escapement and carry it for part-placing; FR1.2.2: Drive the intermittent temporized transfer; FR3.2.1: Materialize the escapement gateway; FR3.2.2: Actuate the gateway mechanism.

The following design parameters (DPs) of third order have been found:

DP1.1.1: Linear gravity feed track; DP1.1.2: Curvilinear delivery chute (with vertical accumulating end); DP1.2.1: Rotary disk feeder (with circularly disposed slots); DP1.2.2: Driving kinematic chain; DP3.2.1: Gate flaps; DP3.2.2: Rotating electromagnets.

The last level (the fourth) of decomposition for FRs consists of:

FR1.2.2.1: Provide driving power; FR1.2.2.2: Provide flexible transmission; FR 1.2.2.3: Provide speed de-multiplication; FR 1.2.2.4: Translate continuous rotation into intermittent temporized rotation; FR1.2.2.5: Protect against indexing blockage.

Respectively to those, the following design parameters (DPs) have been identified:

DP1.2.2.1: Electrical $24 \mathrm{~V}$ c.c. motor; DP1.2.2.2: Belt transmission; DP1.2.2.3: Gear transmission; DP1.2.2.4: Maltese cross (Geneva) mechanism; DP1.2.2.5: Clutch with balls and springs.

In Table 1, the Master Design Matrix corresponding for the considered uncoupled design solution is presented and it can be observed that the result is a diagonal matrix.

The way approached in defining the FRs, the decomposition process and the way the mapping for DPs was accomplished, all of these under the rules of FRs independence and of information minimizing, have been found as essential in obtaining such a final result. 
Table 1. Master design matrix resulting for the considered uncoupled design solution.

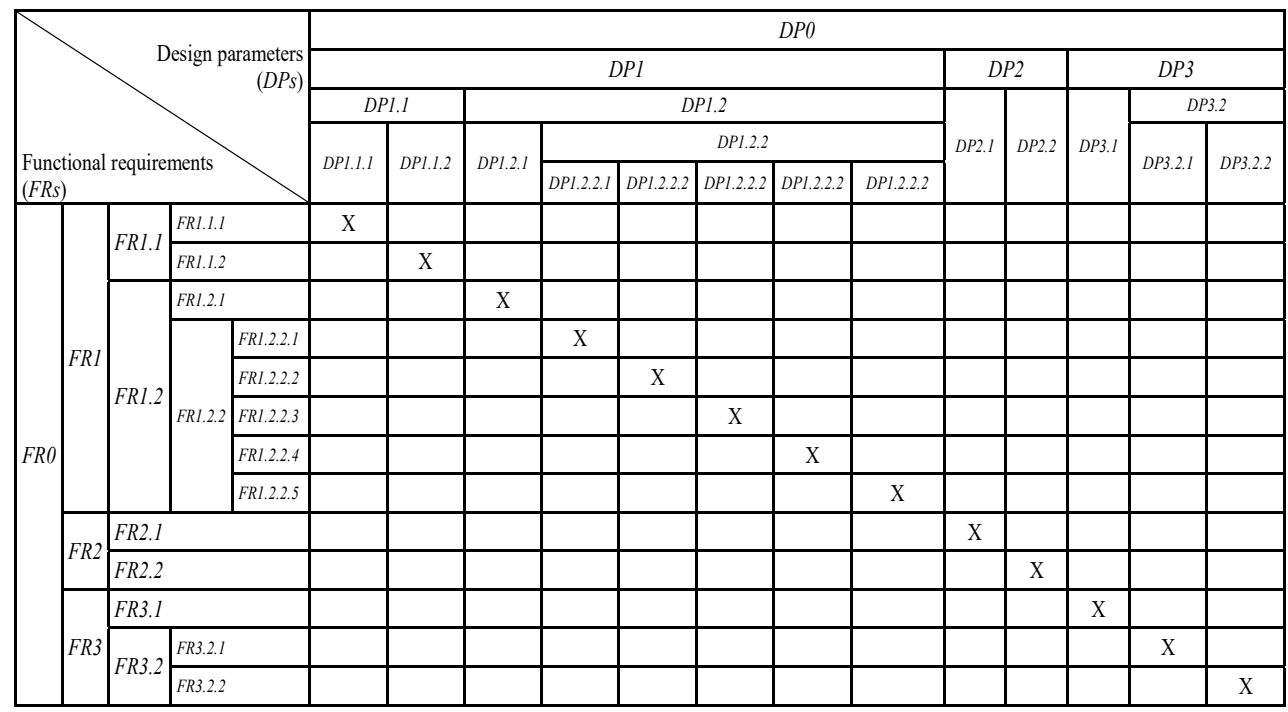

\section{Results. 3D parameterized model for the mechanical structure}

In Figure 3, the 3D parameterized model for the mechanical structure of the automated sorting system is presented as a deliverable result of the case study. The model has been developed within an educational project, [25], using advanced capabilities of Solid Edge as $\mathrm{CAD} / \mathrm{CAE}$ tool [26]. In Figure 3, the above mentioned DPs are identified. The decomposition model obtained by applying AD theory has delivered a reliable modular FRs/DPs based product structure, which provided a good solution for the 3D parametric product model structure, in terms of capturing the Design Intent. More than that, it has streamlined the CAD activities by eliminating iterative trials in defining the CAD model structure.

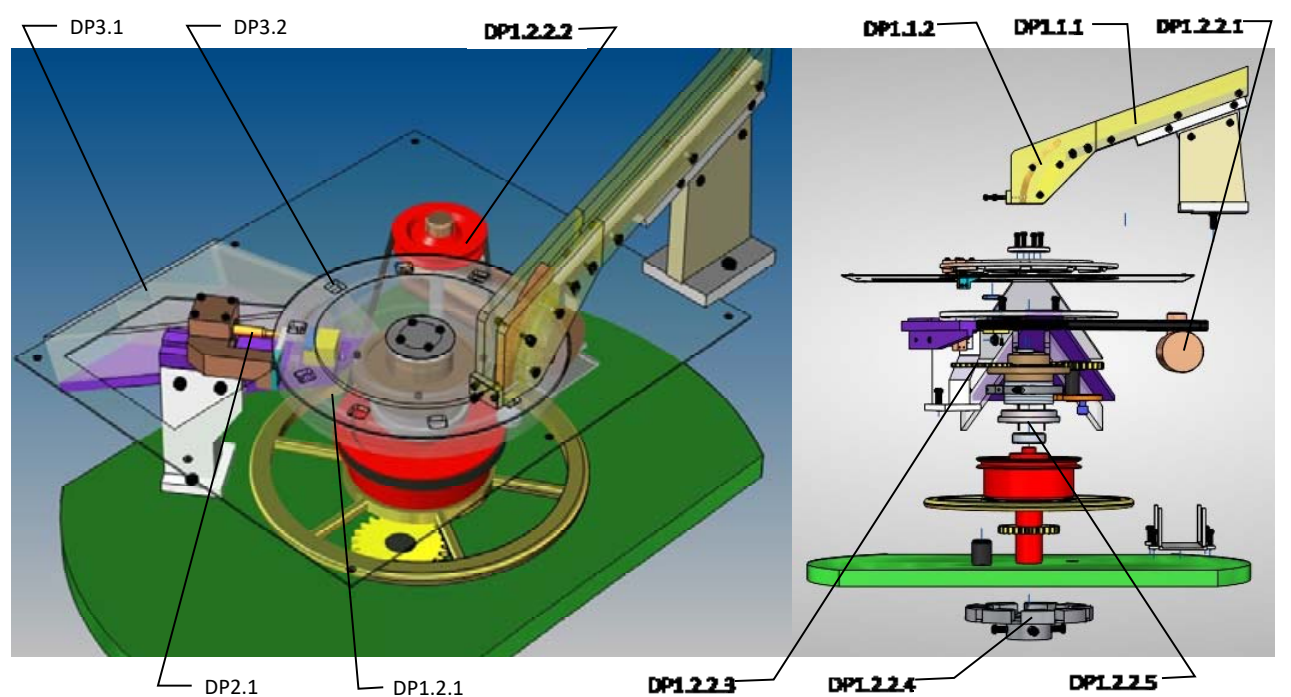

Fig. 3. Mechanical structure of the automated sorting system - parameterized 3D model (after [25]). 


\section{Conclusions}

In addition to those said at the end of the previous subtitles, we can say, as a general conclusion, that the results of the case study have confirmed the two above mentioned research hypotheses. Streamlining of the CAD modelling activities can be achieved by convergent use of $\mathrm{AD}$ theory together with some $\mathrm{DfX}$ principles and tools, but the AD model behavior depends on correctly defining the FRs and on the decomposition approach rules (simplicity and independence). Researches have to extend the involvement of AD theory within Detailed Design stages, considering an in-depth analysis of NPD process.

\section{References}

1. N.P. Suh, The Principles of Design (Oxford University Press, New York, 1990)

2. N.P. Suh, Axiomatic Design: Advances and Applications (Oxford University Press, New York, 2001)

3. N.P. Suh, Complexity: Theory and Applications (Oxford University Press, New York, 2005)

4. C.A. Brown, Proceedings of the $21^{\text {st }}$ CIRP Design Conference, 173-175 (2011)

5. M. Kressy, Industrial Design (Rhode Island School of Design, Designturn Inc., 2002)

6. I.M. Pista et al., MATEC Web of Conferences 137, 04006 (2017)

7. I.M. Pista et al., Bulletin of the Polytechnic Institute of Iasi, Machine Construction 63(67) no.3, 9-20 (2017)

8. G. Nagit et al., MATEC Web of Conferences 127, 01021 (2017)

9. V. Merticaru jr. et al., MATEC Web of Conferences, 112, 03008 (2017)

10. A.M. Del Corral, J. Fernández, M. González, X. Riudor, Temes de disseny 32, 81-97 (2016)

11. K. Scharwath, Yves Béhar's 7 Principles of Holistic Product Design (Triple Pundit, 2011)

12. A.S.Yigit, A.G. Ulsoy, A. Allahverdi, Journal of Intelligent Manufacturing 13, 309316 (2002)

13. Y. Koren, M. Shpitalni, JMSY 29, 130-141 (2010)

14. G.Q. Huang, Design for X: Concurrent engineering imperatives (Springer Science, 1996)

15. V. Merticaru jr., M.I. Ripanu, Applied Mechanics and Materials 371, 499-503 (2013)

16. A.M. Mihalache et al., Applied Mechanics and Materials 657, 725-729 (2014)

17. G. Musca, A. Mihalache, L. Tabacaru, IOP Conf. Ser.: Mater. Sci. Eng. 161, 012019 (2016)

18. V. Merticaru jr., Automation and Robotics in Technological Processes (in Romanian) (PIM, Iasi, 2008)

19. Tr. Gramescu, O. Lupescu, Automation of Technological Processes - Laboratory applications (in Romanian) (Iasi Polytechnic Institute, 1990)

20. G. Boothroyd, Assembly Automation and Product Design, (CRC Press, 1991)

21. V. Merticaru, E. Merticaru, Mechanisms (in Romanian) (VIE, Iasi, 2002)

22. V. Merticaru, E. Merticaru, MOCM (JESR) 1, 1389 (1995)

23. P. Crowther, Design for disassembly - Themes and principles (RAIA/BDP Environment Design Guide, 2005)

24. M.M. Cucos et al., Bulletin of the Polytechnic Institute of Iasi, Machine Construction 63(67) no.3, 21-40 (2017)

25. I. Butnariu, Bachelor Thesis (TUIASI, 2017)

26. G. Musca, L. Tabacaru, Performance in design with Solid Edge ST and technical data management in product development (in Romanian) (PIM, Iasi, 2010) 